\title{
Nonlinear interfacial motion in magnetohydrodynamic flows
}

\section{Chihiro Matsuoka, Katsunobu Nishihara, Takayoshi Sano}

\begin{tabular}{|c|l|}
\hline Citation & High Energy Density Physics, 31; 19-23 \\
\hline Issue Date & $2019-04$ \\
\hline Type & Journal Article \\
\hline Textversion & author \\
\hline Rights & $\begin{array}{l}\text { C2019 Elsevier B.V. This manuscript version is made available under the } \\
\text { CC-BY-NC-ND 4.0 License. https://creativecommons.org/licenses/by-nc-nd/4.0/ } \\
\text { This is the accepted manuscript version. The article has been published in final form } \\
\text { at https://doi.org/10.1016/j.hedp.2019.02.002 }\end{array}$ \\
\hline DOI & 10.1016/j.hedp.2019.02.002 \\
\hline
\end{tabular}

Self-Archiving by Author(s)

Placed on: Osaka City University Repository 


\title{
Nonlinear interfacial motion in magnetohydrodynamic flows
}

\author{
Chihiro Matsuoka $^{a, *}$, Katsunobu Nishihara ${ }^{a, b}$, Takayoshi Sano $^{b}$ \\ ${ }^{a}$ Laboratory of Applied Mathemetics, Graduate School of Engineering, Osaka City \\ University, Sugimoto-cho, Sumiyoshi, Osaka 558-8585, Japan \\ ${ }^{b}$ Institute of Laser Engineering, Osaka University, Suita, Osaka 565-0871, Japan
}

\begin{abstract}
Nonlinear motion of vortex sheets with non-uniform current is investigated taking the magnetohydrodynamic Richtmyer-Meshkov instability (MHD RMI) and the magnetohydrodynamic Kelvin-Helmholtz instability (MHD KHI) as the examples. As the ratio of the magnetic force to the convective force increases, Alfvén oscillations appear and the nonlinear growth of the interface as a vortex sheet is suppressed. We show that the turbulent energy possessing the interface flows into the magnetic energy, which causes the strong magnetic field amplification for both instabilities. We also discuss the difference of the temporal evolution of the interface between MHD RMI and MHD KHI.
\end{abstract}

Keywords: current-vortex sheet, MHD Richtmyer-Meshkov instability, MHD Kelvin-Helmholtz instability

\section{Introduction}

When a supernova explosion occurs, the materials that composed the star scatter in high speeds accompanied by strong shock waves, and these distributed materials, called supernova remnants (SNR's) expand into space and finally become sources of new solar systems. Giacalone et al. [1], Uchiyama et al. [2], and Inoue et al. [3] have determined that SNR's possess a strong magnetic field compared with the surrounding interstellar medium, and they

Email address: matsuoka@mech.eng.osaka-cu.ac.jp (Chihiro Matsuoka ${ }^{a, *}$ ) 
concluded that a turbulent region with a locally strong magnetic field is expected to be spread out in areas of frequent supernova explosions, such as those at the galactic center and starburst galaxies. Using ideal MHD simulations, Sano et al. concluded that the stretch of the interface in the RichtmyerMeshkov instability (RMI) $[4,5,6]$ in magnetohydrodynamic (MHD) flows causes magnetic field amplification in an SNR [7], and the amplification or suppression of the magnetic field in an SNR depends on the Mach number of the incident shock [8]. These works suggest that the interfacial motion in MHD flows is essential for the magnetic field amplification.

The behaviors of hydromagnetic interfaces, including Alfvén surface waves, have been previously studied [9, 10, 11]; Chen and Hasegawa determined a coupled wave solution for the Kelvin-Helmholtz instability (KHI) and the shear Alfvén wave [11]. Chandrasekhar studied convective motion in a magnetic field and concluded that the convection is inhibited by the magnetic field [12]. This suggests that the vortex motion such as RMI and KHI is suppressed by the existence of a magnetic field. All these works consider the linear stability of the fluid interface. Taking into account the plasma sheath region and introducing the thickness of a current-vortex sheet, Arshukova et al. have theoretically discussed the deformation of the interface [13]. Hunter and Thoo performed a weakly nonlinear analysis and derived an amplitude equation to describe the propagation of a wave on a current-vortex sheet [15]. Müller and Grappin have investigated MHD turbulences using spectral DNS and concluded that field-line deformation by turbulent motions leads to magnetic field amplification [16]. The former analytical studies do not consider the large deformation of the interface, and the role of vortex sheets in the magnetic field amplification is unclear in the latter DNS results.

Despite the significance of vortex sheets in astrophysics and plasma physics, very few theoretical models have been proposed in the investigation of their nonlinear motion in MHD flows. Extending the concept of a vortex sheet $[17,18,19]$, Matsuoka et al. proposed a theoretical model to describe the magnetic field amplification mechanism such as observed in SNR's using MHD-RMI [20]. The sharp density jump in RMI induces two different magnetic fields across the interface, and the tangential discontinuities of these quantities induce a non-uniform current-vortex sheet in MHD-RMI. Unlike the classical current sheets that appear, for instance, in magnetic reconnection processes in space plasmas $[21,22,23]$, the current sheets considered in MHD RMI are non-uniform and non-stationary current-vortex sheets. The sheet model presented in this study is compatible with the analytical stud- 
ies described above $[9,10,11,12,15]$, and it allows easy calculation of the various geometrical quantities of the interface such as the stretch rate $[7,20]$ and curvature, which are important factors contributing to the magnetic field amplification.

It is reported in some studies that the magnetic field has a suppressing effect on the growth of fluid instabilities $[12,8,24]$. The suppression of fluid instabilities is significantly important in MHD turbulences [25, 26], ICF, and ISM. In the current study, applying the current-vortex sheet model developed in the reference [20], we investigate the suppressing effect of the magnetic field in MHD RMI and MHD KHI, and discuss the difference in the evolution of the interfaces between MHD RMI and MHD KHI.

This paper is organized as follows. In Section 2, we present the governing equations and briefly review for the current-vortex sheet model. In Section 3, we provide numerical results by the sheet model and discuss the effect of the magnetic field on the interface in MHD RMI. We also compare the temporal evolution of the interfaces in MHD RMI and MHD KHI, and discuss the difference between the two. Section 4 is devoted to conclusion.

\section{Governing equations and overview of current-vortex sheet model}

We consider the two-dimensional inviscid and incompressible magnetohydrodynamic flows. Then the governing equations are given by

$$
\begin{aligned}
& \frac{\partial \boldsymbol{u}}{\partial t}+(\boldsymbol{u} \cdot \nabla) \boldsymbol{u}-\frac{1}{\rho \mu}(\boldsymbol{B} \cdot \nabla) \boldsymbol{B}=-\frac{1}{\rho} \nabla\left(p+\frac{\boldsymbol{B} \cdot \boldsymbol{B}}{2 \mu}\right), \\
& \frac{\partial \boldsymbol{B}}{\partial t}=\nabla \times(\boldsymbol{u} \times \boldsymbol{B}), \\
& \nabla \cdot \boldsymbol{u}=0 \\
& \nabla \cdot \boldsymbol{B}=0 \\
& \mu \boldsymbol{j}=\nabla \times \boldsymbol{B},
\end{aligned}
$$

where $\boldsymbol{u}$ denotes the fluid velocity related with the velocity potential $\phi$ as $\boldsymbol{u}=\nabla \phi, \boldsymbol{B}$ the magnetic field, $\boldsymbol{j}$ the current density, $\rho$ the mass density, $p$ the fluid pressure, and $\mu$ the permeability. We set $\mu=1$ in the following.

We consider a fluid interface with tangential velocity jump. This sharp density jump induces two different valued magnetic fields $\boldsymbol{B}_{1}$ and $\boldsymbol{B}_{2}$ and velocities $\boldsymbol{u}_{1}$ and $\boldsymbol{u}_{2}$ between the interface, where the upper fluid (fluid 2; 
$\rho=\rho_{2}$ ) is assumed to be equal or lighter than the lower one (fluid $1 ; \rho=\rho_{1}$ ). Due to the existence of the tangential discontinuities of the fluid velocity and the magnetic field, this interface becomes a current-vortex sheet. We assume that the bulk is current free; i.e., $\boldsymbol{j}=0$ in the bulk. This assumption leads to $\boldsymbol{\omega}=\nabla \times \boldsymbol{u}=0$ in the bulk [20]. These two conditions guarantee that the current localizes only on the interface (i.e., the current does not flow in the bulk; this condition is true if and only if the initial magnetic field is imposed to be parallel to the initial interface).

We parameterize points on the interface as

$$
\boldsymbol{X}(\theta, t)=[X(\theta, t), Y(\theta, t)]
$$

using a Lagrangian parameter $\theta$ that parametrizes the interface. We define the velocity $\boldsymbol{u}^{+}$of a Lagrangian point labelled by $\theta$ as

$$
\boldsymbol{u}^{+}(\theta, t)=\boldsymbol{q}+\frac{\tilde{\alpha} \gamma}{2} \boldsymbol{t}
$$

where $\boldsymbol{q} \equiv(U, V)=\left(\boldsymbol{u}_{1}+\boldsymbol{u}_{2}\right) / 2, \boldsymbol{\gamma}=\boldsymbol{u}_{1}-\boldsymbol{u}_{2}$, and $\gamma=\boldsymbol{\gamma} \cdot \boldsymbol{t}=\partial \Gamma / \partial s$ denotes the vortex sheet strength derived from the circulation at the interface $\Gamma \equiv \phi_{1}-\phi_{2}\left(\boldsymbol{u}_{i}=\nabla \phi_{i}, \phi_{i}\right.$ the velocity potential $)$, in which $s$ and $\boldsymbol{t}$ are length and unit tangent of the interface, respectively. Here, $\tilde{\alpha}$ is an artificial parameter depending on the Atwood number $A=\left(\rho_{2}-\rho_{1}\right) /\left(\rho_{1}+\rho_{2}\right)$ and it relates to how to select the tangential velocity $[17,19]$.

Equating $\boldsymbol{u}^{+}$with the evolution of the interface [17], we have the interfacial velocity for the Lagrangian motion as

$$
\frac{\mathrm{d} X}{\mathrm{~d} t}=U+\frac{\tilde{\alpha} X_{\theta}}{2 s_{\theta}} \gamma, \quad \frac{\mathrm{d} Y}{\mathrm{~d} t}=V+\frac{\tilde{\alpha} Y_{\theta}}{2 s_{\theta}} \gamma
$$

where

$$
\frac{\mathrm{d}}{\mathrm{d} t}=\frac{\partial}{\partial t}+\boldsymbol{u}^{+} \cdot \nabla
$$

is the Lagrangian derivative in the frame moving with the interface. The subscript $\theta$ denotes the differentiation with respect to $\theta$ and $s_{\theta}=\sqrt{X_{\theta}^{2}+Y_{\theta}^{2}}$.

The average velocity $\boldsymbol{q}$ can be rewritten by the complex form $q^{*}=U-i V$. Then we obtain the vortex induced velocity due to the Biot-Savart law [29]:

$$
q^{*}=U-i V=\frac{1}{2 \pi i} \mathrm{PV} \int_{-\infty}^{\infty} \frac{\gamma\left(\theta^{\prime}\right) s_{\theta}\left(\theta^{\prime}\right) d \theta^{\prime}}{z(\theta)-z\left(\theta^{\prime}\right)}
$$


where PV denotes the principal value. Equation (7) is called the BirkhoffRott equation $[27,28]$ and it provides the velocity of a vortex sheet.

Under the above conditions, integrating Eq. (1) along a narrow rectanglar region between the interface and taking the limit of thickness zero for the interface, we obtain the evolution equation for the circulation $\Gamma[20]$ :

$$
\frac{\mathrm{d} \Gamma}{\mathrm{d} t}=2 A \frac{\mathrm{d} \Phi}{\mathrm{d} t}-A \boldsymbol{q} \cdot \boldsymbol{q}+\frac{A+2 \tilde{\alpha}}{4} \boldsymbol{\gamma} \cdot \boldsymbol{\gamma}-\tilde{\alpha} A \boldsymbol{\gamma} \cdot \boldsymbol{q}+\frac{2}{\rho_{1}+\rho_{2}}\langle\boldsymbol{B}\rangle \cdot \boldsymbol{j}_{s},
$$

where $\Phi=\left(\phi_{1}+\phi_{2}\right) / 2,\langle\boldsymbol{B}\rangle=\left(\boldsymbol{B}_{1}+\boldsymbol{B}_{2}\right) / 2, \boldsymbol{j}_{s}=\boldsymbol{B}_{1}-\boldsymbol{B}_{2}$ denotes the surface current density, and $j_{s}=\boldsymbol{j}_{s} \cdot \boldsymbol{t}$ provides the current sheet strength.

Differentiating (8) with respect to $\theta$, we obtain the following Fredholm integral equation of the second kind:

$$
\begin{aligned}
\frac{\mathrm{d} \gamma}{\mathrm{d} t} & =\frac{2 A}{s_{\theta}}\left(X_{\theta} \frac{\mathrm{d} U}{\mathrm{~d} t}+Y_{\theta} \frac{\mathrm{d} V}{\mathrm{~d} t}\right)-\frac{(1+\tilde{\alpha} A) \gamma}{s_{\theta}^{2}}\left(X_{\theta} U_{\theta}+Y_{\theta} V_{\theta}\right) \\
& +\frac{A+\tilde{\alpha}}{4 s_{\theta}}\left(\gamma^{2}\right)_{\theta}+\frac{1}{s_{\theta} R_{A}^{2}}\left(\langle\boldsymbol{B}\rangle \cdot \boldsymbol{j}_{s}\right)_{\theta},
\end{aligned}
$$

where $R_{A}$ is defined by

$$
R_{A} \equiv \sqrt{\frac{\rho_{1}+\rho_{2}}{2}} \frac{v_{l i n}}{B_{0}}=\frac{v_{l i n}}{v_{a 0}},
$$

in which $v_{a 0}$ denotes the Alfvén velocity given by

$$
v_{a 0}^{2}=\frac{2 B_{0}^{2}}{\rho_{1}+\rho_{2}}
$$

using the initial homogeneous magnetic field $B_{0}=|\boldsymbol{B}|_{t=0}$ and $v_{\text {lin }}$ is a quantity having the dimension of the velocity. For the case of MHD RMI, this $v_{l i n}$ is provided as the initial velocity shear of the pure RMI:

$$
v_{\text {lin }}=\frac{\rho_{1} \delta v_{1}^{*}-\rho_{2} \delta v_{2}^{*}}{\rho_{1}+\rho_{2}}
$$

in which $\delta v_{1,2}^{*}$ represent the transverse velocities immediately after the shockinterface interaction $[6,30,31]$, while it is given as the velocity of the initial main stream $[18,29]$ for MHD KHI. The parameter $R_{A}$ is a quantity that measures the magnitude of the Lorentz force $\boldsymbol{j} \times \boldsymbol{B}=1 / \mu\left(\boldsymbol{B} \cdot \nabla \boldsymbol{B}-1 / 2 \nabla B^{2}\right)$ in the Euler equation (1) and it is called the Alfvén (Mach) number. 
In order to amplify the magnetic field, the Alfvén wave needs to propagate parallel to the interface. This indicates that the magnetic field $\boldsymbol{B}$ does not have a finite $y$ component at $y= \pm \infty$. Otherwise, the Alfvén wave is apart from the interface, and the magnetic field cannot receive the fluid energy from the vortex sheet. Then the magnetic field amplification does not occur [8].

We can prove that $[20]$

$$
\frac{\mathrm{d}}{\mathrm{d} t}\left(\boldsymbol{B}_{i} \cdot \boldsymbol{n}\right)=0
$$

where $\boldsymbol{n}$ is the unit normal to the interface. This equation indicates that if the initial magnetic field is applied parallel to the interface, the normal component of the magnetic field does not occur even for $t>0$. Then we have only to consider the evolution of the tangential component of the magnetic field and the induction equation (2) can be rewritten as

$$
\begin{aligned}
& \frac{\mathrm{d} B_{1}^{t}}{\mathrm{~d} t}=\frac{\tilde{\alpha}-1}{2 s_{\theta}} \gamma B_{1, \theta}^{t}+\frac{B_{1}^{t}}{s_{\theta}}\left(q_{\theta}^{t}+\frac{\gamma_{\theta}}{2}\right), \\
& \frac{\mathrm{d} B_{2}^{t}}{\mathrm{~d} t}=\frac{\tilde{\alpha}+1}{2 s_{\theta}} \gamma B_{2, \theta}^{t}+\frac{B_{2}^{t}}{s_{\theta}}\left(q_{\theta}^{t}-\frac{\gamma_{\theta}}{2}\right),
\end{aligned}
$$

where

$$
B_{i, \theta}^{t}=\frac{\partial B_{i}^{t}}{\partial \theta} \quad(i=1,2), \quad q_{\theta}^{t}=\frac{\partial}{\partial \theta}(\boldsymbol{q} \cdot \boldsymbol{t}) .
$$

Solving the evolution equations (6), (7), (9), and (12) simultaneously, we can determine the motion of a current-vortex sheet.

\section{Numerical results by the sheet model}

In this section, we present some numerical results of equations (6), (7), (9), and (12) solved by using the vortex method [17, 19, 20]. We set the initial magnetic field as $\boldsymbol{B}_{t=0} \equiv \boldsymbol{B}_{0}=B_{0} \boldsymbol{t}$, and use the normalizations $k \boldsymbol{x} \rightarrow \boldsymbol{x}$ and $\boldsymbol{B}_{i} / B_{0} \rightarrow \boldsymbol{B}_{i}$ for both MHD RMI and MHD KHI, where $k$ is the wave number. Furthermore, we adopt the following normalizations $k v_{l i n} t \rightarrow t$, $k \Gamma / v_{\text {lin }} \rightarrow \Gamma, \gamma / v_{\text {lin }} \rightarrow \gamma$ for MHD RMI, while $k U_{0} t \rightarrow t, k \Gamma / U_{0} \rightarrow \Gamma$, $\gamma / U_{0} \rightarrow \gamma$ for MHD KHI, where the constant $U_{0}=O(1)$ is the main stream in KHI.

When we calculate the MHD RMI, we select the Atwood numbers $A=$ -0.2 and $A=0$ ( $A \leq 0$ in our choice), while we select $A=0$ only for the 
calculations of MHD KHI. The artificial parameter $\tilde{\alpha}$ is chosen as $\tilde{\alpha}=-A^{2}$ and $\tilde{\alpha}=0$ for the calculation of $A=-0.2$ and $A=0$, respectively. The grid points $N$ is set to $N=1024$ for all calculations and the regularized parameter $\delta$ [18] is selected as $\delta=0.15$. For details of the numerical method, refer to the reference [20].

The initial condition for MHD RMI is set to

$$
x(\theta, 0)=\theta, \quad y(\theta, 0)=0 ; \quad \gamma(\theta, 0)=-2 \sin \theta,
$$

while the initial condition for MHD KHI is

$$
x(\theta, 0)=\theta+a_{0} \sin \theta, \quad y(\theta, 0)=-a_{0} \sin \theta ; \quad \gamma(\theta, 0)=2,
$$

where the initial amplitude $a_{0}$ is selected as $a_{0}=0.1$. The initial homogeneous magnetic field is set to

$$
\boldsymbol{B}_{j}(\theta, 0)=\hat{\boldsymbol{e}_{x}}, \quad(j=1,2)
$$

for both MHD RMI and MHD KHI, where $\hat{\boldsymbol{e}_{x}}$ is the unit vector in the $x$ direction.

Figure 1 shows the interfacial shape at the fully nonlinear stage in MHD RMI with various the Alfvén numbers, where the Atwood number $A=-0.2$. The Alfvén number $R_{A}=\infty$ corresponds to the Pure RMI. As the Alfvén number decreases, i.e., as the effect of the Lorentz force increases, the RMI is suppressed, and the Alfvén oscillation appears. This Alfvén oscillation is called the surface Alfvén wave $[10,11]$. When $R_{A}^{2} \leq 0.1$, the interfacial motion is almost linear, and the growth of the amplitude is not found. This result suggests that the fluid instabilities can be suppressed by a magnetic field, which indicates that the turbulent energy is transformed into the magnetic energy [8, 20].

We show the difference in the interfacial evolution between MHD RMI and MHD KHI in Fig. 2. When $R_{A}^{2}=\infty$; i.e., for the pure RMI and pure KHI, the strength of the roll-up is the same; however, as the value of the Alfvén number decreases, the difference between the two instabilities becomes evident. When $R_{A}^{2}=10$, the roll-up of the interface is not found in MHD RMI, on the other hand, the interface in MHD KHI preserves the property as a vortex sheet even in this Alfvén number. This suggests that MHD RMI is easier to control by the magnetic field than MHD KHI.

Figure 3 shows the temporal evolution of the normalized maximum magnetic field $\left(R_{A}^{-1}|\boldsymbol{B}| /\left|B_{0}\right|\right)^{2}$, maximum vortex sheet strength $|\gamma|$, and maximum stretching rate of the interface for various Alfvén numbers in MHD RMI 

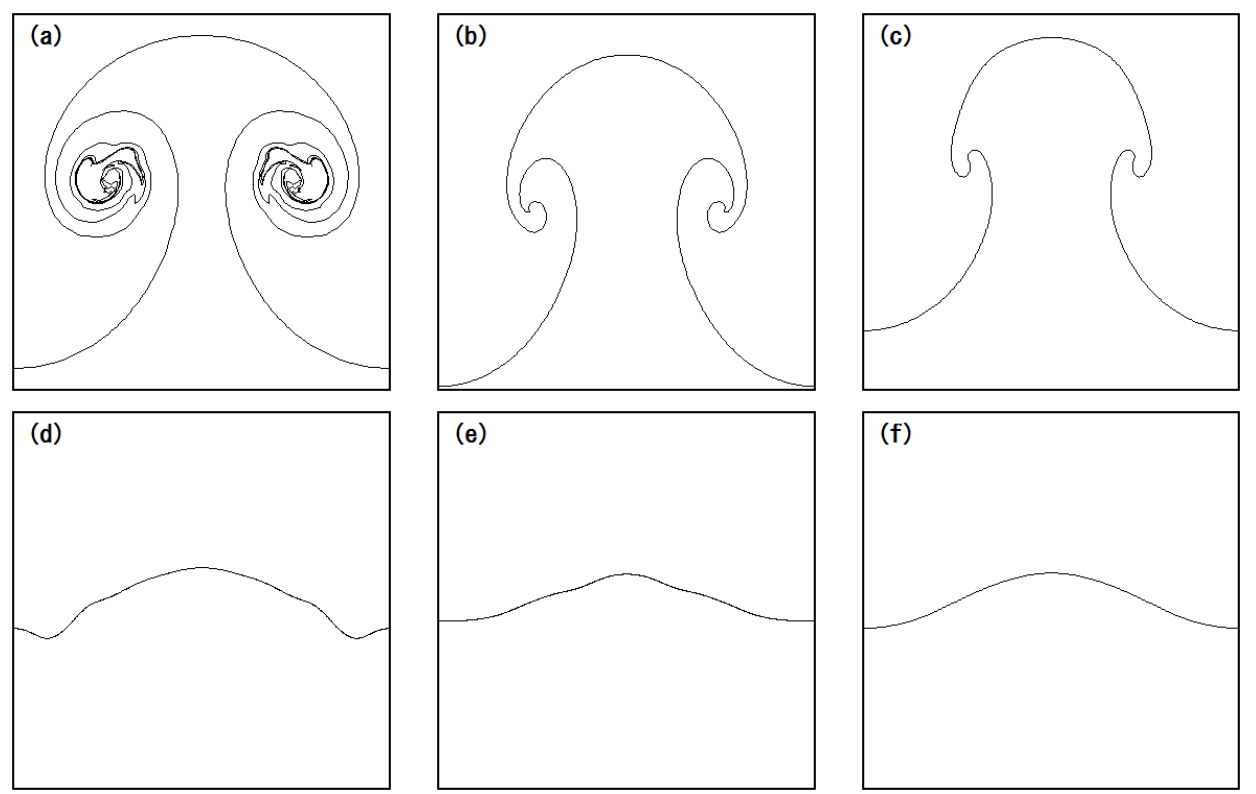

Figure 1: Difference in the evolution of the interface by changing the Alfvén number, where $R_{A}^{2}=(\mathrm{a}) \infty$ (b) 1000 (c) 100 (d) 10 (e) 1 (f) 0.1 . 

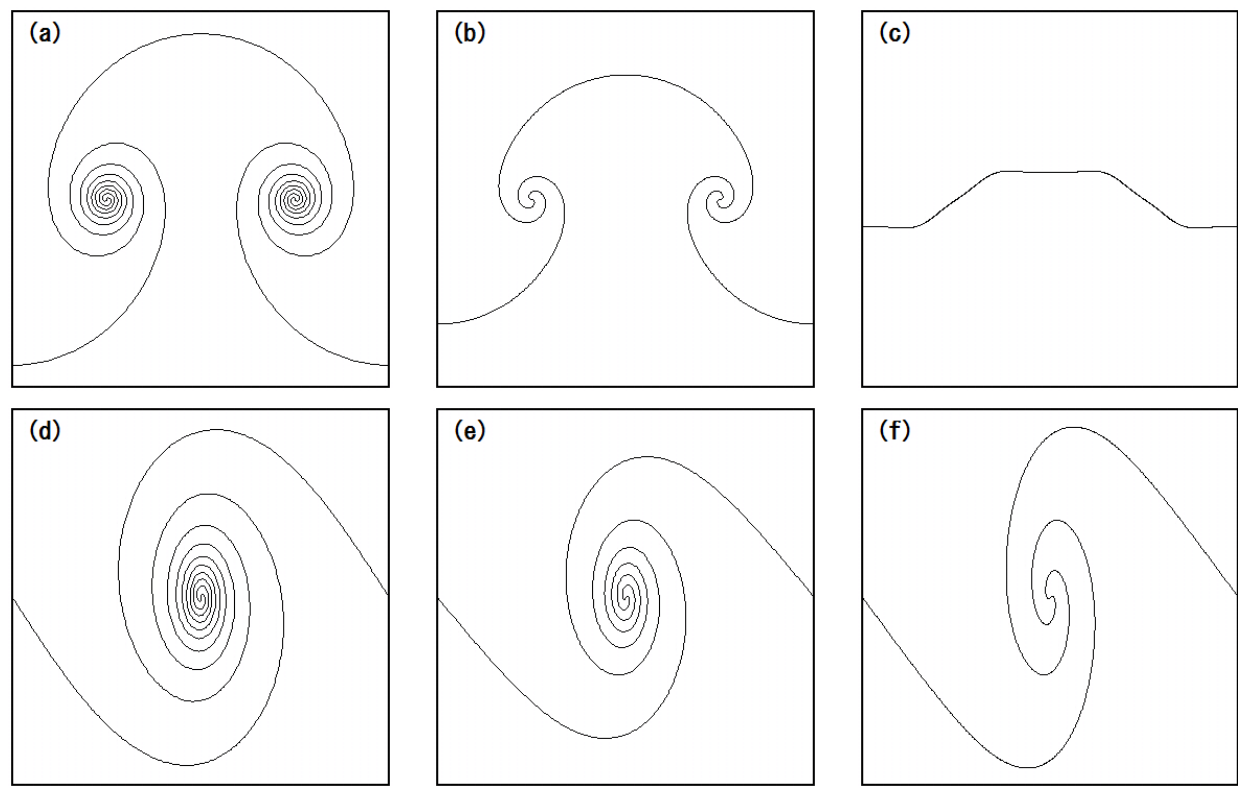

Figure 2: Difference in the evolution of the interface between MHD RMI (a) - (c) and MHD KHI (d) - (f), where the Alfvén number $R_{A}^{2}=$ (a) \& (d) $\infty$, (b) \& (e) 1000, and (c) $\&$ (f) 10. The Atwood number $A$ is set to $A=0$ for all figures. 

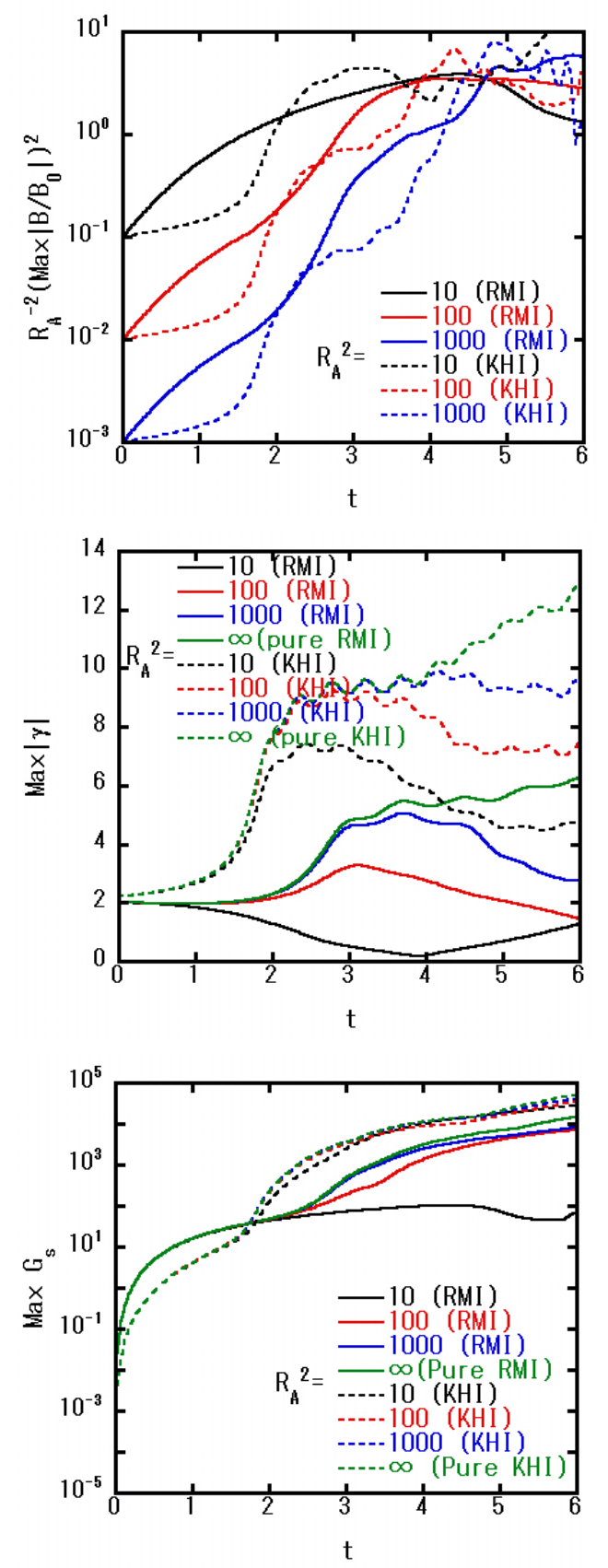

Figure 3: Temporal evolution of the maximum magnetic field (upper figure), maximum vortex sheet strength (middle figure), and maximum stretching rate (lower figure) for MHD RMI and MHD KHI $(A=0)$. 
and MHD KHI. The sheet strength $|\gamma|$ is a quantity providing the magnitude of the kinetic energy of a vortex sheet. These maximum values appear at the vortex cores (the center of the roll-up) for both MHD RMI and MHD KHI when $A=0$. The values of the maximum magnetic field attain almost the same value of $O(1)$ irrelevant to $R_{A}$, and then from the definition (10), $v_{\text {lin }} \sim v_{a 0}\left(U_{0} \sim v_{a 0}\right.$ in MHD KHI) holds at this point. This suggests that the kinetic energy of turbulence; i.e., the energy with a vortex sheet is transformed into the magnetic energy and the magnetic field amplification is saturated $[7,8,20]$. We can also find this consideration in the figure of the maximum vortex sheet strength, in which we see that the maximum $|\gamma|$ decreases as the Alfvén number decreases. As we see from the figure, the energy of the vortex sheet in KHI is larger than that in RMI, which is also reflected in Fig. 2, where the interface in MHD KHI preserves the property as a vortex sheet even for small Alfvén numbers. We mention that when $t<1.3$, which corresponds to the linear stage in KHI, the roll-up of the interface is not found, and the magnetic field amplification is weak in MHD KHI at this stage.

The stretching rate of the interface $G_{s}(t)$ is defined by [20]

$$
G_{s}(t)=\int \frac{\partial\left(\boldsymbol{u}_{2} \cdot \boldsymbol{t}\right)}{\partial s} \mathrm{~d} t .
$$

When $A=0$, we can replace $\boldsymbol{u}_{2}$ in this equation with $\boldsymbol{u}_{1}$. The stretching rate $G_{s}(t)$ corresponds to the leading term in the evolution equation for the magnetic field (12) derived from the induction equation (2). The stretching rate is a quantity that measures the geometrical growth of an interface into the tangential direction. We see that as the value of $G_{s}$ becomes large, the magnetic field at the interface increases [except the case of $R_{A}^{2}=10$ in MHD RMI, in which the interface does not grow, see Fig. 2 (c)].

\section{Conclusion}

We have presented a theoretical model to describe the fully nonlinear motion of current-vortex sheets in MHD RMI and MHD KHI. We found that the kinetic energy of the vortex sheet is transformed into the magnetic energy and the magnetic field suppresses these fluid instabilities. When we compare MHD RMI and MHD KHI with the same Alfvén number, MHD RMI can be suppressed by a smaller magnetic field. This fact is important when we consider the control of fluid instabilities in ICF and ISM because 
it is difficult to generate a large magnetic field experimentally. We can also calculate physical quantities in bulk such as the magnetic field, the velocity field, and so on by use of the sheet model presented here for the case that the bulk vorticity does not exist [20]. If the normal component to the interface exists in the initial magnetic field, the current-vortex sheet approximation would not be valid, and we should consider the other analytical treatment for the nonlinear evolution.

\section{Acknowledgements}

This work was supported by Grant-in-Aid for Scientific Research (C) (Grant No. 17K05371) from the Japan Society for the Promotion of Science and the joint research project of ILE, Osaka University.

\section{References}

[1] J. Giacalone, J. R. Jokipii, Magnetic field amplification by shocks in turbulent fluids, Astrophys. J. 663 (2007) L41-44.

[2] Y. Uchiyama, F. A. Aharonian, T. Tanaka, T. Takahashi, Y. Maeda, Extremely fast acceleration of cosmic rays in a supernova remnant, Nature 449 (2007) 576-578.

[3] T. Inoue, J. Shimoda, Y. Ohira, R. Yamazaki, The origin of radially aligned magnetic fields in young supewrnova remnants, Astrophys. J. 772 (2012) L20-24.

[4] R. D. Richtmyer, Taylor instability in shock acceleration of compressible fluids, Commun. Pure Appl. Math. 13 (1960) 297-319.

[5] E. E. Meshkov, Instability of the interface of two gases accelerated by a shock wave, Sov. Fluid Dynamics 4 (1969) 101-108.

[6] K. Nishihara, J. G. Wouchuk, C. Matsuoka, R. Ishizaki, V. V. Zhakhovskii, Richtmyer-Meshkov instability: theory of linear and nonlinear evolution, Phil.Trans. Roy. Soc. A 368 (2010) 1769-1807 (references therein). 
[7] T. Sano, K. Nishihara, C. Matsuoka, T. Inoue, Magnetic field amplification associated with the Richtmyer-Meshkov instability, Astrophys. J. 758 (2012) 126.

[8] T. Sano, T. Inoue, K. Nishihara, Critical magnetic field strength for suppression of the Richtmyer-Meshkov instability in plasmas, Phys. Rev. Lett. 111 (2013) 205001.

[9] M. Kruskal, M. Schwarzschild, Some Instabilities of a Completely Ionized Plasma, Proc. R. Soc. Lond. A 223 (1954) 348-360.

[10] R. Gerwin, Hydromagnetic surface waves in a conducting liquid surrounded by a compressible gas, Phys. Fluids 10 (1967) 2164-2170.

[11] L. Chen, A. Hasegawa, A Theory of long period magnetic pulsations, 2. Impulse excitation of surface eigen-modes, J. Geophys Res. 79 (1974) 1033-1037.

[12] S. Chandrasekhar, On the inhibition of convection by a magnetic field, Phil. Mag. 43 (1952) 501-532.

[13] I. L. Arshukova, V. E. Nikolai, H. K. Biernat, Magnetohydrodynamic instability of a high magnetic shear layer with a finite curvature radius, Phys. Plasmas 9 (2002) 401-408.

[14] K. L. Ilin, Y. L. Trakhinin, V. A. Vladimirov, The stability of steady magnetohydrodynamic flows with current-vortex sheets, Phys. Plasmas 10 (2003) 2649-2658.

[15] J. K. Hunter, J. B. Thoo, On the weakly nonlinear Kelvin-Helmholtz instability of tangential discontinuities in MHD, J. Hyper. Diff. Eqs. 8 (2011) 691-726.

[16] W. C. Müller, R. Grappin, Spectral energy dynamics in magnetohydrodynamic turbulence, Phys. Rev. Lett. 95 (2005) 114502.

[17] G. Baker, D. I. Meiron, S. A. Orszag, Generalized vortex methods for free surface flow problems, J. Fluid Mech. 123 (1982) 477-501.

[18] R. Krasny, Computation of vortex sheet roll-up in the Trefftz plane, J. Fluid Mech. 184 (1987) 123-155. 
[19] C. Matsuoka, K. Nishihara, Vortex core dynamics and singularity formations in incompressible Richtmyer-Meshkov instability, Phys. Rev. E 73 (2006) 026304, 74 049902(E).

[20] C. Matsuoka, K. Nishihara, T. Sano, Nonlinear Dynamics of Nonuniform Current-Vortex Sheets in Magnetohydrodynamic Flows, J. Nonlinear Sci. 27 (2017) 531-572.

[21] E. R. Priest, The magnetohydrodynamics of current sheets, Rep. Prog. Phys. 48 (1985) 955-1090 (references therein).

[22] J. J. Aly, T. Amari, Current sheets in two-dimensional potential magnetic fields I. General properties, Astron. Astrophys. 221 (1989) 287-294.

[23] S. E. Gibon, F. Bagenal, B. C. Low, Current sheets in the solar minimum corona, J. Geo. Res. 101 (1996) 4813-4824.

[24] V. Wheatley and R. Samtaney and D. I. Pullin, R. M. Gehre, The transverse field Richtmyer-Meshkov instability in magnetohydrodynamics, Phys. Fluids, 26 (2014) 016102.

[25] W. C. Muller, R. GrappinA, Spectral energy dynamics in magnetohydrodynamic turbulence, Phys. Rev. Lett. 95 (2005) 114502.

[26] A. Alexakis, Large-Scale Magnetic Fields in Magnetohydrodynamic Turbulence, Phys. Rev. Lett. 110 (2013) 084502.

[27] G. Birkhoff, Helmholtz and Taylor instability, Proc. Symp. Appl. Maths. Soc. 13 (1962) 55-76.

[28] N. Rott, Diffraction of a weak shock with vortex generation, J. Fluid Mech. 1 (1956) 111-128.

[29] P. G. Saffman, Vortex dynamics, Cambridge University Press, Cambridge (1992).

[30] J. G. Wouchuk, K. Nishihara, Linear perturbation growth at a shocked interface, Phys. Plasmas 3 (1996) 3761-3776.

[31] J. G. Wouchuk, K. Nishihara, Asymptotic growth in the linear Richtmyer-Meshkov instability, Phys. Plasmas 4 (1997) 1028-1038. 